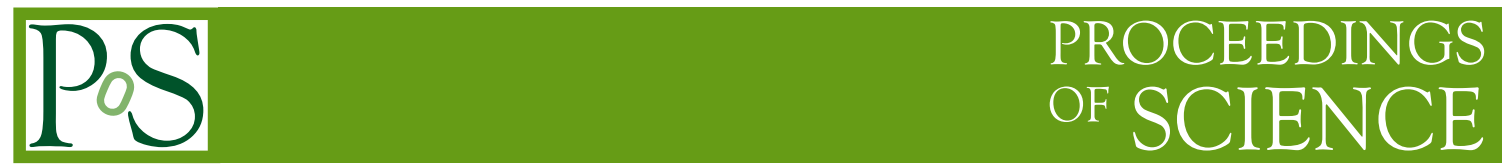

\title{
Chiral low-energy constants from lattice QCD
}

\section{Silvia Necco*}

CERN, Physics Departement, 1211 Geneva 23, Switzerland

E-mail: Silvia.Neccodcern.ch

Different strategies for the computation of QCD low-energy couplings by matching lattice results with the chiral effective theory are reviewed. After recalling some relevant predictions from the effective theory, the current status of leading order $\left(\Sigma, F, \Sigma_{0}, F_{0}\right)$ and next-to-leading order $\left(l_{i}, L_{i}\right)$ low-energy constants is summarized, focusing on recent results obtained with $N_{f}=2$ and $N_{f}=2+1$ lattice simulations.

8th Conference Quark Confinement and the Hadron Spectrum

September 1-6 2008

Mainz, Germany

*Work partially supported by EC Sixth Framework Program under the contract MRTN-CT-2006-035482 (FLAVIAnet), by the Ministerio de Ciencia e Innovación under Grant No. FPA2007-60323 and by CPAN (Grant No. CSD2007-00042). Preprint numbers: IFIC/08-62, CERN-PH-TH-2009-015. 


\section{Introduction}

The dynamics of QCD at low momenta can be described in terms of an effective theory, which encodes the spontaneous breaking of chiral symmetry

$$
\mathrm{SU}\left(N_{f}\right)_{L} \times \mathrm{SU}\left(N_{f}\right)_{R} \rightarrow \mathrm{SU}\left(N_{f}\right)_{V}
$$

$N_{f}$ is the number of light quark flavours; we will consider both cases $N_{f}=2,3$. The effective Lagrangian in Euclidean space can be expanded in powers of momenta as [1-3]

$$
\mathscr{L}_{\chi}=\mathscr{L}_{\chi}^{(2)}+\mathscr{L}_{\chi}^{(4)}+\cdots
$$

where

$$
\begin{aligned}
& \mathscr{L}_{\chi}^{(2)}=\frac{F^{2}}{4} \operatorname{Tr}\left[\partial_{\mu} U^{\dagger} \partial_{\mu} U\right]-\frac{\Sigma}{2} \operatorname{Tr}\left[\mathscr{M} U+U^{\dagger} \mathscr{M}^{\dagger}\right], \\
& \mathscr{L}_{\chi}^{(4)}=\sum_{i} C_{i} \mathscr{O}_{i} .
\end{aligned}
$$

$U \in \mathrm{SU}\left(N_{f}\right)$ represents the pseudo Nambu-Goldstone bosons degrees of freedom, and $\mathscr{M}$ is the $\left(N_{f} \times N_{f}\right)$ quark mass matrix. The low-energy dynamics is parametrised by the so-called LowEnergy-Couplings (LECs) of the effective theory, which are not determined by symmetries. At leading order, the chiral Lagrangian involves two LECs, namely the pseudoscalar decay constant $F$ and the chiral condensate $\Sigma$ (or equivalently $B=\Sigma / F^{2}$ ) ${ }^{1}$. At next-to-leading order, $\mathscr{L}_{\chi}^{(4)}$ contains 10 terms for the case $N_{f}=2$ and 12 for $N_{f}=3$, with corresponding couplings

$$
\begin{aligned}
& \left\{C_{i} \rightarrow l_{i=1 . .7}, h_{i=1 . .3} \quad\left(N_{f}=2\right),\right. \\
& \left\{C_{i} \rightarrow L_{i=1 . .10}, H_{i=1 . .2} \quad\left(N_{f}=3\right) .\right.
\end{aligned}
$$

Once the LECs are fixed, chiral perturbation theory becomes a predictive framework and hence a powerful tool for understanding the phenomenology of QCD at low dynamics. A review of recent applications has been presented at this conference by G. Ecker [4]. Those couplings must be ideally computed from"first principles": from this point of view lattice QCD is a promising approach, since -once the physical point has been reached (i.e. continuum, infinite volume limit and physical light quark masses)-it does not introduce any model dependence.

For some decades lattice simulations have been performed far from the chiral limit and introducing simplifications such as quenching. Chiral effective theory has been then extensively used to understand the results of lattice QCD, in particular to guide extrapolations to the chiral limit and to estimate the volume dependence of physical observables computed on the lattice. In the past years lattice computations experienced an important progress, due to an interplay of new theoretical developments, algorithmic improvements and increasing powerful computing resources. The effect of this progress is that now lattice unquenched simulations with $N_{f}=2$ and $N_{f}=2+1$ light dynamical flavours are approaching domains where a reliable matching with the chiral effective theory can be performed. Reliable in this case means that light quark masses are as close as possible to the physical value, and that different sources of systematic errors can be at least in principle taken under control, namely finite-volume effects, lattice artifacts and renormalisation.

\footnotetext{
${ }^{1}$ In the following $F, \Sigma$ and $B$ will refer to the case $N_{f}=2$; for $N_{f}=3$ the notation $F_{0}, \Sigma_{0}, B_{0}$ will be used.
} 


\section{Low-Energy couplings from quark-mass dependence of pseudoscalar masses and decay constants}

The LECs can be extracted for instance by studying the quark-mass dependence of given observables in lattice QCD and match it with the predictions of the chiral effective theory. In this section I will summarize some of these predictions, in particular for the pseudoscalar decay constants and masses. The up and down quarks are clearly sufficiently light to formulate a SU(2) $\times \mathrm{SU}(2)$ chiral effective theory where pions are treated as pseudo Nambu-Goldstone bosons and Kaons and etas are integrated out. In this theory, the LECS will depend on the higher energy scales $m_{s}, m_{c}, m_{b}, \Lambda_{\mathrm{QCD}}$. Alternatively, one can decide to treat also the strange quark as light and formulate a $\mathrm{SU}(3) \times \mathrm{SU}(3)$ effective theory, where also Kaons and etas are treated as pseudo Nambu-Goldstone bosons and the LECs will not depend on $m_{s}$ anymore.

One of the simplest but deepest predictions which can be formulated at low energy is the so-called Gell-Mann-Oakes-Renner relation [5], which states that the square of the pion mass is linearly proportional to the light quark mass:

$$
M_{\pi}^{2}=M^{2}=2 \hat{m} B
$$

where $\hat{m}=\left(m_{u}+m_{d}\right) / 2$. This corresponds to the leading order prediction in the effective theory. Higher order corrections can be computed systematically. In particular for the $\mathrm{SU}(2) \times \mathrm{SU}(2)$ theory one finds the NLO expressions [2]

$$
\begin{aligned}
M_{\pi}^{2} & =M^{2}+\frac{M^{4}}{32 \pi^{2} F^{2}} \ln \left(\frac{M^{2}}{\Lambda_{3}^{2}}\right), \\
F_{\pi} & =F-\frac{M^{2}}{16 \pi^{2} F^{2}} \ln \left(\frac{M^{2}}{\Lambda_{4}^{2}}\right),
\end{aligned}
$$

where $\Lambda_{3,4}$ are related to the scale-independent NLO LECs $\bar{l}_{3,4}$ by the relation

$$
\bar{l}_{3,4} \equiv \ln \left(\frac{\Lambda_{3,4}^{2}}{M^{2}}\right)_{M=139.6 \mathrm{MeV}} .
$$

By matching the quark-mass dependence of $F_{\pi}$ and $M_{\pi}$ with lattice results, it is hence possible to extract the LO LECs $F$ and $\Sigma$ (or equivalently $B$ ) and the NLO couplings $\bar{l}_{3}, \bar{l}_{4}$.

In this theory there is the possibility to treat Kaons as external matter fields coupled to pions, in analogy to what is done in the heavy-light meson chiral perturbation theory. The quark mass dependence of Kaon quantities can then be worked out explicitly in this framework [6].

In the $\mathrm{SU}(3) \times \mathrm{SU}(3)$ theory, the quark mass dependence of pion and Kaon masses and decay constants is given by [3]

$$
\begin{aligned}
M_{\pi}^{2} & =2 \hat{m} B_{0}\left\{1+\mu_{\pi}-\frac{1}{3} \mu_{\eta}+2 \hat{m} K_{3}+K_{4}\right\}, \\
M_{K}^{2} & =\left(\hat{m}+m_{s}\right) B_{0}\left\{1+\frac{2}{3} \mu_{\eta}+\left(\hat{m}+m_{s}\right) K_{3}+K_{4}\right\}, \\
F_{\pi} & =F_{0}\left\{1-2 \mu_{\pi}-\mu_{K}+2 \hat{m} K_{6}+K_{7}\right\}, \\
F_{K} & =F_{0}\left\{1-\frac{3}{4} \mu_{\pi}-\frac{3}{2} \mu_{K}-\frac{3}{4} \mu_{\eta}+\left(\hat{m}+m_{s}\right) K_{6}+K_{7}\right\},
\end{aligned}
$$


with

$$
\mu_{P}=\frac{M_{P}^{2}}{32 \pi^{2} F_{0}^{2}} \ln \left(\frac{M_{P}^{2}}{\mu^{2}}\right)
$$

The $K_{i}$ contain the NLO LECs:

$$
\begin{array}{cc}
K_{3}=\frac{8 B_{0}}{F_{0}{ }^{2}}\left(2 L_{8}-L_{5}\right), & K_{4}=\left(m_{u}+m_{d}+m_{s}\right) \frac{16 B_{0}}{F_{0}^{2}}\left(2 L_{6}-L_{4}\right), \\
K_{6}=\frac{4 B_{0}}{F_{0}{ }^{2}}\left(L_{5}\right), \quad K_{7}=\left(m_{u}+m_{d}+m_{s}\right) \frac{8 B_{0}}{F_{0}{ }^{2}}\left(L_{4}\right) .
\end{array}
$$

In this case, the matching with lattice results allows the extraction of $F_{0}, \Sigma_{0}\left(B_{0}\right), L_{4}, L_{5},\left(2 L_{8}-\right.$ $\left.L_{5}\right),\left(2 L_{6}-L_{4}\right)$. Moreover, the $m_{s}$-dependence of the $\mathrm{SU}(2) \times \mathrm{SU}(2)$ LECs can be explicitly worked out [3]

$$
\begin{aligned}
& F=F_{0}\left\{1+8 \frac{\bar{M}_{K}^{2}}{F_{0}^{2}} L_{4}-\bar{\mu}_{K}+O\left(m_{s}^{2}\right)\right\}, \\
& \Sigma=\Sigma_{0}\left\{1+32 \frac{\bar{M}_{K}^{2}}{F_{0}^{2}} L_{6}-2 \bar{\mu}_{K}-\bar{\mu}_{\eta}+O\left(m_{s}^{2}\right)\right\},
\end{aligned}
$$

where $\bar{M}_{K}$ is the Kaon mass in the limit $m_{u}, m_{d} \rightarrow 0, \bar{M}_{K}^{2}=m_{s} B_{0}$. In the large- $N_{c}$ limit one finds $\Sigma / \Sigma_{0}, F / F_{0}, B / B_{0} \rightarrow 1$, which corresponds to the so-called Okubo-Zweig-Iizuka (OZI) rule. Paramagnetic inequalities can be invoked to predict the sign of the deviations from the OZI rule, $\left(F / F_{0}-1\right)>0,\left(\Sigma / \Sigma_{0}-1\right)>0[7]$

Lattice simulations are performed on finite boxes with volume $V=L^{3} T$, and finite-volume effects may play a relevant role. An important point is that chiral perturbation theory can be used also to infer information about finite-size scaling of physical observables [8-10]. In the asymptotic region $M_{\pi} L \gg 1$, the dominant effect is of the form $e^{-M_{\pi} L}$. The finite-size effects for the pion mass have been computed at two loops [11]; alternatively, resummations of asymptotic Lüscher formulas [12] for pseudoscalar masses and decay constants have been investigated [13].

Lattice calculations are often performed in so-called partially quenched setup, where $m_{\text {sea }} \neq$ $m_{v a l}$. It is possible to extend the effective theory formalism to this case and obtain predictions from partially quenched chiral perturbation theory [14] which can be matched with the lattice results.

Finally, lattice simulations are performed at small but finite lattice spacing $a$; ideally one should first perform a continuum extrapolation of lattice results and then match with the chiral effective theory. In alternative, one can formulate the chiral effective theory at finite lattice spacing by adopting the Symanzik formalism $[15,16]$. The price to pay is the appearing of extra couplings in the effective theory which have to be determined by the matching. Notice that for particular discretizations, where flavour symmetry is broken at finite lattice spacing, for example for the socalled staggered fermions with fourth root prescription, the limits $a \rightarrow 0$ and $m \rightarrow 0$ have to be taken simultaneously. Lattice results must be then matched with the so-called staggered chiral perturbation theory [17].

In several recent lattice studies with $N_{f}=2$ dynamical fermions the quark mass dependence of $M_{\pi}$ and $F_{\pi}$ has been matched with $\mathrm{SU}(2) \times \mathrm{SU}(2)$ chiral perturbation theory in order to extract $F, \Sigma$, $\bar{l}_{3}, \bar{l}_{4}$. The relevant parameters of these simulations are summarised in Table 1: the discretization 


\begin{tabular}{ccccc}
\hline Collaboration & Dirac operator & $a(\mathrm{fm})$ & $M_{\pi}(\mathrm{MeV})$ & $M_{\pi, \min } L$ \\
\hline CERN-TOV [19] & Wilson $+O(a)$ impr. & $0.052-0.072\left(M_{K}\right)$ & $\gtrsim 380$ & $3.2-3.6$ \\
ETM [20, 21] & Wilson TM & $0.065-0.1\left(F_{\pi}\right)$ & $\gtrsim 265$ & $3.2-3.6$ \\
JLQCD/TWQCD [22] & Neuberger & $0.12\left(r_{0}\right)$ & $\gtrsim 290$ & 2.9 \\
\hline
\end{tabular}

Table 1: Simulation parameters of recent $N_{f}=2$ computations.

\begin{tabular}{ccccc}
\hline Collaboration & Dirac operator & $a(\mathrm{fm})$ & $M_{\pi}(\mathrm{MeV})$ & $M_{\pi, \min } L$ \\
\hline MILC [23, 24] & Staggered & $0.06-0.18\left[F_{\pi}\right]$ & $\gtrsim 240$ & 4 \\
NPLQCD [25] & Domain Wall + Staggered & $0.13\left[r_{0}\right]$ & $\gtrsim 290$ & 3.7 \\
RBC/UKQCD [26] & Domain Wall & $0.11\left[M_{\Omega}\right]$ & $\gtrsim 330$ & 4.6 \\
PACS-CS [27] & Wilson $+O(a)$ impr. & $0.09\left[M_{\Omega}\right]$ & $\gtrsim 160$ & 2.3 \\
\hline
\end{tabular}

Table 2: Simulation parameters of recent $N_{f}=2+1$ computations. $r_{0}$ is the so-called Sommer scale [28].

adopted, the lattice spacing (in parenthesis the quantity that has been used to fix the scale), the pseudoscalar meson masses and the volume in terms of the quantity $M_{\pi} L$.

One of the main outcomes of these studies is that $M_{\pi}^{2}$ is nearly a linear function of $\hat{m}$, as predicted by the GMOR relation, up to relatively large masses (of the order of $m_{s} / 2$ ). A further common conclusion is that for $M_{\pi} \lesssim 450 \mathrm{MeV}$ lattice results seem compatible with prediction of the chiral effective theory at NLO. CERN-TOV and JLQCD pointed out however that for $F_{\pi}$ NNLO effects may be significant and this could affect the systematic uncertainty on the determination of $\bar{l}_{4}$.

Several collaborations are performing lattice calculations with $2+1$ dynamical flavours, i.e. with two light (degenerate) quarks and one heavier quark (which is usually fixed at the physical strange mass $m_{s}$ ). In analogy to the $N_{f}=2$ case, we report the relevant simulation parameters in Table $2^{2}$. The MILC data have been analysed using SU(3) $\times$ SU(3) (partially quenched) rooted staggered chiral perturbation theory including analytic NNLO and NNNLO terms. The NPLQCD collaboration adopted Domain Wall valence quarks and staggered sea quarks, and fitted the data using continuum chiral perturbation theory at NLO and (partial) NNLO. RBC/UKQCD adopted a partially quenched setup, while PACS-CS used only unitary points. These two collaborations matched their results for pseudoscalar masses and decay constants with $\mathrm{SU}(3) \times \mathrm{SU}(3)$ effective theory at NLO and observed poor convergence of the perturbative series around the physical strange quark mass. On the other hand, the NLO SU(2) $\times \mathrm{SU}(2)$ analysis seems to yield more reliable results.

The results obtained for the LECs are all summarized in the Tables 3, 4, 5, 6 .

\section{Other approaches to determine LECs on the lattice}

\subsection{LECs from $\varepsilon$-regime simulations}

On a finite volume $V=L^{3} T$ with $L \gg 1 / \Lambda_{\mathrm{QCD}}$, different chiral regimes can be distinguished.

\footnotetext{
${ }^{2}$ Another collaboration has recently published results for hadron masses obtained with $2+1$ lattice simulations [18].
} 
Approaching the chiral limit by keeping $M_{\pi} L \gg 1$ (like already mentioned in Section 2) defines the so-called $p$-regime, where finite-volume effects are exponentially suppressed, while mass-effects are dominant. Alternatively, one can approach the chiral limit by keeping $\mu=m \Sigma V \lesssim O(1)$; this defines the so-called $\varepsilon$-regime $[8,9]$, where the Compton wavelength associated to the NambuGoldstone bosons is larger than the linear extent of the box, $M_{\pi} L<1$. In this case the power counting is reorganised such that mass effects are suppressed, while finite-volume effects are enhanced and become polynomial in $L^{-2}$. One of the consequences of the rearrangements is that, at a given order in the effective theory, less LECs appear with respect to the $p$-regime, and the predictions are less "contaminated" by higher order unknown couplings. For instance, the NLO predictions of given correlation functions contain only the LO couplings: $F, \Sigma\left(F_{0}, \Sigma_{0}\right)$ can then be extracted by matching the finite-size scaling of correlators computed on the lattice with the predictions of the effective theory.

As an example, the pseudoscalar and axial correlators at NLO can be written as (for $N_{f}=2$ ) [29]

$$
\begin{aligned}
& C_{P}(t)=\frac{1}{L^{3}} \int d^{3} \vec{x}\langle P(x) P(0)\rangle=\Sigma^{2}\left[a_{P}+\frac{T}{F^{2} L^{3}} b_{P} h_{1}\left(\frac{t}{T}\right)\right], \\
& C_{A}(t)=\frac{1}{L^{3}} \int d^{3} \vec{x}\left\langle A_{0}(x) A_{0}(0)\right\rangle=\frac{F^{2}}{V}\left[a_{A}+\frac{T}{F^{2} L^{3}} b_{A} h_{1}\left(\frac{t}{T}\right)\right],
\end{aligned}
$$

where $h_{1}(\tau)=\frac{1}{2}\left[\left(\tau-\frac{1}{2}\right)^{2}-\frac{1}{12}\right]$, and $a_{P}, b_{P}, a_{A}, b_{A}$ are dimensionless functions of $\mu, L, T$. Furthermore, in the $\varepsilon$-regime topology plays a relevant role [30]: observables may be defined at fixed value of the topological charge, and the dependence on this charge should be also reproduced by the chiral effective theory.

This strategy has been applied recently in two studies. JLQCD [31] computed mesonic correlation functions at fixed topology in the $\varepsilon$-regime using the Neuberger Dirac operator with $N_{f}=2$ dynamical quarks. A. Hasenfratz and collaborators [32] adopted improved Wilson fermions combined with the reweighting technique. The results obtained for $F$ and $\Sigma$ are reported in the summary Table $3(8,9)$. The two-point functions computed in [32] have been recently reanalysed in [33] including the leading $O\left(a^{2}\right)$ correction inferred via the so-called Wilson chiral perturbation theory applied to the $\varepsilon$-regime [33, 34], finding small corrections with respect to the continuum results.

Two-point mesonic functions have been computed in the effective theory also in the case of non-degenerate quark masses, and in particular in the case where some quarks are in the $p$-regime and others in the $\varepsilon$-regime [35-37]. This may offer new possibilities to extract the LECs from lattice data.

\subsection{LECs from eigenvalues distribution}

At $\mathrm{LO}$ in the $\varepsilon$-expansion, the partition function is equivalent to the one of a chiral Random Matrix Theory (RMT) [38-41]; it follows that RMT reproduces the same microscopic spectral density $\rho_{S}(\zeta, \mu)$ of the chiral effective theory, in terms of two dimensionless variables $\zeta=\lambda \Sigma V$ and $\mu=m \Sigma V$, where $\lambda$ represents the eigenvalues of the Dirac operator. Moreover, one can extract the probability distributions of single eigenvalues [42-44]; hence it is possible to match the QCD low-lying spectrum of the Dirac operator $\left\langle\lambda_{k}\right\rangle^{\mathrm{QCD}}$ with the expectation values $\left\langle\zeta_{k}\right\rangle^{\mathrm{RMT}}$ (where $k=1,2 .$. labels the eigenvalues) in order to extract the chiral condensate $\Sigma$. This method has been 
applied by many authors; the results obtained by the JLQCD/TWQCD collaboration [45] an by DeGrand and collaborators [46] are reported in Table $3(10,11)$.

This framework can be extended such that the spectrum of the Dirac operator is sensitive also to the pseudoscalar decay constant $F$ at LO in the effective theory [47]. This strategy has been adopted by DeGrand and collaborators [48], yielding the results reported in Table 3 (12).

In another recent work [49] it is shown that the quark condensate can be extracted from suitable (renormalizable) spectral observables defined in the $p$-regime, for instance the number of Dirac operator modes contained in a given interval. The method has been used on Wilson lattice QCD and the result for the condensate is also given in Table 3 (6). In correlation to this, an interesting development from the side of the effective theory has been investigated in [50], where the quark condensate and the spectral density of the Dirac operator have been computed by using a technique which is able to smoothly connect $p$ - and $\varepsilon$-regimes.

\subsection{LECs from form factors}

The pion electromagnetic form factor, defined as

$$
\left\langle\pi^{+}\left(p^{\prime}\right)\left|V_{\mu}\right| \pi^{+}(p)\right\rangle=\left(p+p^{\prime}\right)_{\mu} F_{V}^{\pi \pi}\left(q^{2}\right)
$$

with $q^{2}=-Q^{2}=\left(p-p^{\prime}\right)^{2}$, can be matched with the predictions of the chiral effective theory. In particular, at NLO it turns out to be related to the LEC $l_{6}$ for the $\mathrm{SU}(2) \times \mathrm{SU}(2)$ theory [2] and to $L_{9}$ in the $\mathrm{SU}(3) \times \mathrm{SU}(3)$ case [51]. The pion electromagnetic form factor has been computed on the lattice and compared with chiral effective theory by the RBC/UKQCD collaboration [52] and by the ETM collaboration [53], using the lattice parameters already given in Tables 1,2. The latter performed a NNLO chiral fit, which -by using as input the experimental value of the scalar pion radius- allowed the extraction of $F, \Sigma, \bar{l}_{1}, \bar{l}_{2}, \bar{l}_{3}, \bar{l}_{4}, \bar{l}_{6}$. We report some of the results in Tables 3,5. Moreover, an exploratory study of the scalar form factor has been presented by the JLQCD/TWQCD collaboration at the last lattice conference [54].

\section{Summary and Conclusions}

In Tables 3, 4, 5, 6 we summarize the results for the low-energy couplings obtained through lattice simulations.

In particular, Table 3 collects the results for the LO LECs, both from $p$-regime and $\varepsilon$-regime studies. The first error is statistical, while the following uncertainties (if present) are systematic. Although errors are still quite sizeable, the general agreement among the different determinations is reassuring. PACS-CS results for the condensate $\Sigma$ point towards larger values with respect to other collaboration: notice however that in that case perturbative renormalization has been used and systematic errors may be important. Table 4 shows the ratios $F / F_{0}, \Sigma / \Sigma_{0}$; large deviations from 1 would indicate strong violations of the OZI rule. More precise determinations are needed for these observables in order to draw a definitive conclusion.

Table 5 summarizes the results for the $\mathrm{SU}(2) \times \mathrm{SU}(2) \mathrm{NLO}$ constants $\bar{l}_{3}, \bar{l}_{4}, \bar{l}_{6}$. For some of the lattice simulations with $N_{f}=2+1$, we report both values obtained through a direct $\mathrm{SU}(2) \times \mathrm{SU}(2)$ fit and by converting $\mathrm{SU}(3) \times \mathrm{SU}(3)$ into $\mathrm{SU}(2) \times \mathrm{SU}(2)$ LECs by means of chiral perturbation 


\begin{tabular}{|c|c|c|c|c|c|}
\hline Collaboration & $N_{f}$ & $F(\mathrm{MeV})$ & $F_{0}(\mathrm{MeV})$ & $\Sigma^{1 / 3}(\mathrm{MeV})$ & $\Sigma_{0}^{1 / 3}(\mathrm{MeV})$ \\
\hline (1) $\operatorname{ETM}[20,21]$ & 2 & $86.03(5)$ & & $267(2)(9)(4)$ & \\
\hline (2) JLQCD/TWQCD [22] & 2 & $79.0(2.5)(0.7)\left(\begin{array}{l}+4.2 \\
-0.0\end{array}\right)$ & & $235.7(5.0)(2.0)\left(\begin{array}{c}+12.7 \\
-0.0\end{array}\right)$ & \\
\hline (3) MILC [24] & $2+1$ & & & $278(1)\left(\begin{array}{l}+2 \\
-3\end{array}\right)(5)$ & $242(9)\left(\begin{array}{c}+5 \\
-17\end{array}\right)(4)$ \\
\hline (4) RBC/UKQCD [26] & $2+1$ & $81.2(2.9)(5.7)$ & & $255(8)(8)(13)$ & \\
\hline (5) PACS-CS [27] & $2+1$ & $90.3(3.6)$ & $83.8(6.4)$ & $309(7)$ & $290(15)$ \\
\hline (6) Giusti, Lüscher[49] & 2 & & & $276(3)(4)(5)$ & \\
\hline (7) ETM [53] & 2 & $86.6(4)(7)$ & & $264(2)(5)$ & \\
\hline (8) JLQCD[31] & 2 & $87.3(5.6)$ & & $239.8(4.0)$ & \\
\hline (8) A. Hasenfratz et al[32] & 2 & $90(4)$ & & $248(6)$ & \\
\hline (10) JLQCD[45] & 2 & & & $251(7)(11)$ & \\
\hline (11) DeGrand et al[46] & 2 & & & $282(10)$ & \\
\hline (12) DeGrand, Schaefer[48] & 2 & $84(5)$ & & 234(4) & \\
\hline Colangelo, Dürr [58] & phen. & $86.2(5)$ & & & \\
\hline
\end{tabular}

Table 3: Summary of lattice results for LO couplings. The condensate $\Sigma$ is evaluated in the $\overline{\mathrm{MS}}$ scheme at the scale $\mu=2 \mathrm{GeV}$. The upper part (1-7) collects results obtained by matching lattice QCD with the chiral effective theory in the $p$-regime, while the bottom part (8-12) summarizes results obtained in the $\varepsilon$-regime. The last row is the estimation from a phenomenological analysis.

theory. Also in this case the good agreement is encouraging. Lattice data for $\bar{l}_{3}, \bar{l}_{4}$ agree also with phenomenological estimates $[2,55]$. The phenomenological impact of the LECs $\bar{l}_{3}, \bar{l}_{4}$ on the s-wave pion scattering length $a_{0}^{I}(I=0,2)$ has been recently discussed by $\mathrm{H}$. Leutwyler [56].

Finally, Table 6 shows the lattice results for $\mathrm{SU}(3) \times \mathrm{SU}(3) \mathrm{NLO}$ constants $L_{4}, L_{5}$ and the combinations $\left(2 L_{6}-L_{4}\right),\left(2 L_{8}-L_{5}\right)$, at the scale $M_{\rho}=770 \mathrm{MeV}$. These values can be compared with phenomenological estimates reported in [57]. In this case the situation is less clear, since it has been pointed out that $\mathrm{SU}(3) \times \mathrm{SU}(3)$ chiral fits may not be appropriate to describe lattice results obtained with $N_{f}=2+1$. Notice that in order to extract $\mathrm{SU}(3) \times \mathrm{SU}(3)$ couplings, lattice simulations with $N_{f}=3$ light flavours would be more appropriate.

To conclude, important progress occurred in lattice QCD simulation in the past years. Unquenched computations are reaching pion masses as light as $200 \mathrm{MeV}$, lattice spacings around $a \sim 0.07 \mathrm{fm}$ and spatial extends larger than $4 \mathrm{fm}$, moving towards the "physical point". This will allow a precise matching with the chiral effective theory and a deep understanding of low-energy properties of strong interactions. By decreasing systematic uncertainties, systematic errors become a very important issue and have to be carefully studied, namely by controlling the continuum extrapolation, the finite-volume effects, the renormalisation and the uncertainty coming from higher orders in the chiral effective theory. LECs obtained with independent methods and different discretizations tend to point at uniform results within the errors, giving encouraging perspectives for the future.

\section{References}

[1] S. Weinberg, Physica A96 (1979) 327. 


\begin{tabular}{lll}
\hline Collaboration & $F / F_{0}$ & $\Sigma / \Sigma_{0}$ \\
\hline MILC [24] & $1.15(5)\left({ }_{-3}^{+13}\right)$ & $1.52(17)\left({ }_{-15}^{+38}\right)$ \\
RBC/UKQCD [26] & $1.229(59)$ & $1.55(21)$ \\
PACS-CS [27] & $1.065(58)$ & $1.205(14)$ \\
\hline
\end{tabular}

Table 4: Summary of results for the ratios $F / F_{0}$ and $\Sigma / \Sigma_{0}$ obtained in recent $N_{f}=2+1$ lattice simulations.

\begin{tabular}{lllllll}
\hline Collaboration & $N_{f}$ & $\bar{l}_{3}(\mathrm{SU}(2))$ & $\bar{l}_{4}(\mathrm{SU}(2))$ & $\bar{l}_{3}(\mathrm{SU}(3))$ & $\bar{l}_{4}(\mathrm{SU}(3))$ & $\bar{l}_{6}(\mathrm{SU}(2))$ \\
\hline CERN-TOV [19] & 2 & $3.0(5)(1)$ & & & & \\
ETM [20, 21] & 2 & $3.42(8)(10)(27)$ & $4.59(4)(2)(13)$ & & & $14.9(6)(7)$ \\
ETM [53] & 2 & $3.2(4)(2)$ & $4.4(1)(1)$ & & & \\
JLQCD/TWQCD [22] & 2 & $3.44(57)\left({ }_{-68}^{+0}\right)\left({ }_{-0}^{+32}\right)$ & $4.14(26)\left({ }_{-0}^{+49}\right)\left({ }_{-0}^{+32}\right)$ & & & \\
MILC [24] & $2+1$ & & & $1.1(6)\left({ }_{-1.5}^{+1.0}\right)$ & $4.4(4)\left({ }_{-1}^{+4}\right)$ & \\
RBC/UKQCD [26] & $2+1$ & $3.13(33)(24)$ & $4.43(14)(77)$ & $2.87(28)$ & $4.10(5)$ & \\
RBC/UKQCD [52] & $2+1$ & & & & & \\
PACS-CS [27] & $2+1$ & $3.14(23)$ & $4.04(19)$ & $3.47(11)$ & $4.21(11)$ & \\
\hline Gasser, Leutwyler [2] & phen. & $2.9(2.4)$ & $4.3(9)$ & & & \\
Colangelo et al [55] & phen. & & $4.4(2)$ & & & \\
\hline
\end{tabular}

Table 5: NLO couplings $\bar{l}_{3}, \bar{l}_{4}, \bar{l}_{6}$ of the $\mathrm{SU}(2) \times \mathrm{SU}(2)$ chiral effective theory. The "SU(2)" label means that the LECs have been extracted directly from a SU(2) fit of lattice data, while the label "SU(3)" indicates the values obtained via the NLO relations which convert SU(3) NLO couplings into the SU(2) ones. The last rows refer to phenomenological estimates.

\begin{tabular}{lllll}
\hline Collaboration & $L_{4} \cdot 10^{3}$ & $L_{5} \cdot 10^{3}$ & $\left(2 L_{6}-L_{4}\right) \cdot 10^{3}$ & $\left(2 L_{8}-L_{5}\right) \cdot 10^{3}$ \\
\hline MILC [24] & $0.1(3)\left(\begin{array}{l}+3 \\
-1\end{array}\right)$ & $1.4(2)\left({ }_{-1}^{+2}\right)$ & $0.3(1)\left(\begin{array}{l}+2 \\
-3\end{array}\right)$ & $0.3(1)(1)$ \\
RBC/UKQCD [26] & $0.14(8)$ & $0.87(10)$ & $0.00(4)$ & $0.24(4)$ \\
PACS-CS [27] & $-0.06(10)$ & $1.45(7)$ & $0.10(2)$ & $-0.21(3)$ \\
NPLQCD [25] & & $1.42(2)\left(\begin{array}{l}+18 \\
-54\end{array}\right)$ & & \\
\hline Bijnens (lat 07) [57] & 0 & 1.46 & 0 & 0.54 \\
\hline
\end{tabular}

Table 6: NLO SU(3) couplings at the scale $M_{\rho}=770 \mathrm{MeV}$ extracted from $N_{f}=2+1$ lattice simulations. In the last line the phenomenological estimate is reported.

[2] J. Gasser and H. Leutwyler, Ann. Phys. 158 (1984) 142.

[3] J. Gasser and H. Leutwyler, Nucl. Phys. B250 (1985) 465.

[4] G. Ecker, (2008), 0812.4196.

[5] M. Gell-Mann, R.J. Oakes and B. Renner, Phys. Rev. 175 (1968) 2195.

[6] A. Roessl, Nucl. Phys. B555 (1999) 507, hep-ph/9904230.

[7] S. Descotes-Genon, L. Girlanda and J. Stern, JHEP 01 (2000) 041, hep-ph/9910537.

[8] J. Gasser and H. Leutwyler, Phys. Lett. B184 (1987) 83.

[9] J. Gasser and H. Leutwyler, Phys. Lett. B188 (1987) 477. 
[10] J. Gasser and H. Leutwyler, Nucl. Phys. B307 (1988) 763.

[11] G. Colangelo and C. Haefeli, Nucl. Phys. B744 (2006) 14, hep-lat/0602017.

[12] M. Luscher, Commun. Math. Phys. 104 (1986) 177.

[13] G. Colangelo, S. Durr and C. Haefeli, Nucl. Phys. B721 (2005) 136, hep-lat/0503014.

[14] S.R. Sharpe and N. Shoresh, Phys. Rev. D62 (2000) 094503, hep-lat/0006017.

[15] K. Symanzik, Nucl. Phys. B226 (1983) 187.

[16] K. Symanzik, Nucl. Phys. B226 (1983) 205.

[17] W.J. Lee and S.R. Sharpe, Phys. Rev. D60 (1999) 114503, hep-lat/9905023.

[18] S. Durr et al., Science 322 (2008) 1224.

[19] L. Del Debbio et al., JHEP 02 (2007) 056, hep-lat/0610059.

[20] ETM, P. Boucaud et al., Phys. Lett. B650 (2007) 304, hep-lat/0701012.

[21] ETM, P. Dimopoulos et al., (2008), 0810.2873.

[22] JLQCD and TWQCD, J. Noaki et al., Phys. Rev. Lett. 101 (2008) 202004, 0806.0894.

[23] MILC, C. Aubin et al., Phys. Rev. D70 (2004) 114501, hep-lat/0407028.

[24] C. Bernard et al., PoS LAT2007 (2007) 090, 0710.1118.

[25] S.R. Beane et al., Phys. Rev. D75 (2007) 094501, hep-lat/0606023.

[26] RBC-UKQCD, C. Allton et al., Phys. Rev. D78 (2008) 114509, 0804.0473.

[27] PACS-CS, S. Aoki et al., (2008), 0807.1661.

[28] R. Sommer, Nucl. Phys. B411 (1994) 839, hep-lat/9310022.

[29] F.C. Hansen, Nucl. Phys. B345 (1990) 685.

[30] H. Leutwyler and A. Smilga, Phys. Rev. D46 (1992) 5607.

[31] JLQCD, H. Fukaya et al., Phys. Rev. D77 (2008) 074503, 0711.4965.

[32] A. Hasenfratz, R. Hoffmann and S. Schaefer, Phys. Rev. D78 (2008) 054511, 0806.4586.

[33] O. Bar, S. Necco and S. Schaefer, (2008), 0812.2403.

[34] A. Shindler, (2008), 0812.2251.

[35] P.H. Damgaard and H. Fukaya, Nucl. Phys. B793 (2008) 160, 0707.3740.

[36] F. Bernardoni and P. Hernandez, JHEP 10 (2007) 033, 0707.3887.

[37] F. Bernardoni et al., JHEP 10 (2008) 008, 0808.1986.

[38] E.V. Shuryak and J.J.M. Verbaarschot, Nucl. Phys. A560 (1993) 306, hep-th/9212088.

[39] J.J.M. Verbaarschot and I. Zahed, Phys. Rev. Lett. 70 (1993) 3852, hep-th/9303012.

[40] J.J.M. Verbaarschot, Phys. Rev. Lett. 72 (1994) 2531, hep-th/9401059.

[41] J.J.M. Verbaarschot and T. Wettig, Ann. Rev. Nucl. Part. Sci. 50 (2000) 343, hep-ph/0003017.

[42] S.M. Nishigaki, P.H. Damgaard and T. Wettig, Phys. Rev. D58 (1998) 087704, hep-th/9803007.

[43] P.H. Damgaard and S.M. Nishigaki, Phys. Rev. D63 (2001) 045012, hep-th/0006111. 
[44] F. Basile and G. Akemann, JHEP 12 (2007) 043, 0710.0376.

[45] H. Fukaya et al., Phys. Rev. D76 (2007) 054503, 0705.3322.

[46] T. DeGrand, Z. Liu and S. Schaefer, Phys. Rev. D74 (2006) 094504, hep-lat/0608019.

[47] G. Akemann et al., Nucl. Phys. B766 (2007) 34, hep-th/0609059.

[48] T. DeGrand and S. Schaefer, Phys. Rev. D76 (2007) 094509, 0708.1731.

[49] L. Giusti and M. Luscher, (2008), 0812.3638.

[50] P.H. Damgaard and H. Fukaya, (2008), 0812.2797.

[51] J. Gasser and H. Leutwyler, Nucl. Phys. B250 (1985) 517.

[52] P.A. Boyle et al., JHEP 07 (2008) 112, 0804.3971.

[53] R. Frezzotti, V. Lubicz and S. Simula, (2008), 0812.4042.

[54] JLQCD, T. Kaneko et al., (2008), 0810.2590.

[55] G. Colangelo, J. Gasser and H. Leutwyler, Nucl. Phys. B603 (2001) 125, hep-ph/0103088.

[56] H. Leutwyler, (2008), 0812.4165.

[57] J. Bijnens, PoS LAT2007 (2007) 004, 0708.1377.

[58] G. Colangelo and S. Durr, Eur. Phys. J. C33 (2004) 543, hep-lat/0311023. 